\title{
Endocarditis infecciosa por enterococo: descripción de 12 casos
}

\author{
P. MARTÍNEZ ODRIOZOLA, J. MUÑOZ SÁNCHEZ ${ }^{1}$ P. ARRIOLA MARTÍNEZ, \\ E. LIZARRALDE PALACIOS, J. M. SANTAMARÍA JÁUREGUI ${ }^{1}$, J. ZUAZO MEABE², \\ R. CISTERNA CÁNCER ${ }^{3}$, F. M. DE LA VILLA
}

Servicios de Medicina Interna, ${ }^{1}$ Enfermedades Infecciosas, ${ }^{2}$ Cirugía Cardiaca y ${ }^{3}$ Microbiología. Hospital de Basurto. Bilbao

\begin{abstract}
RESUMEN
Con el objetivo de identificar a los pacientes diagnosticados en nuestro hospital de endocarditis infecciosa por enterococo se ha realizado un estudio retrospectivo durante un período de 12 años (1994-2005), analizando sus características clínicas y microbiológicas, así como el tratamiento y la evolución de la enfermedad. Se encontraron 12 casos de endocarditis por enterococo, lo que representó el 6\% del total de endocarditis y el $7 \%$ de las bacteriemias por enterococo. Se trataba de 8 hombres y 4 mujeres, con edades comprendidas entre los 37 y los 94 años. El microorganismo causante fue E. faecalis en 10 casos y E. faecium en 2 casos. Dos de los E. faecalis eran resistentes a aminoglucósidos. La válvula afectada con mayor frecuencia fue la aórtica, con 9 casos, seguida de la mitral, con 2 y de la tricúspide, 1 caso. En 10 pacientes la endocarditis se instaló sobre una válvula nativa y sólo en 2 casos sobre válvula protésica. Únicamente 5 pacientes tenían antecedentes de enfermedad valvular cardiaca. El tratamiento antimicrobiano más utilizado fue la combinación de ampicilina y gentamicina, que se empleó en 8 pacientes. Cuatro casos fueron tratados con monoterapia; 2 de ellos con ampicilina y 2 con vancomicina. Siete pacientes se complicaron con insuficiencia cardíaca, cinco de los cuales precisaron tratamiento quirúrgico de sustitución valvular aórtica. Los 4 enfermos tratados con monoterapia presentaron complicaciones graves, que provocaron la muerte de 2 pacientes.
\end{abstract}

PALABRAS CLAVE: Endocarditis. Enterococcus. Epidemiología.
ENTEROCOCCAL INFECTIVE ENDOCARDITIS: DESCRIPTION OF 12 CASES

\begin{abstract}
We have performed a retrospective study of patients diagnosed with infective endocarditis due to Enterococcus in our hospital for a period of 12 years (1994-2005). We review clinical and microbiological aspects, therapy, and outcome. We found 12 cases that were $6 \%$ of total endocarditis, and $7 \%$ of enterococcal bacteremia. Eight cases were male, and 4 were female, aged between 37 and 94 years. The causative organism of endocarditis was $\mathrm{E}$. faecalis in 10 patients and $\mathrm{E}$. faecium in 2. Two $\mathrm{E}$. faecalis were resistant to aminoglycosides. Aortic valve was the most frequently affected with 9 cases, followed by mitral valve (2 cases), and tricuspid valve (1 case). In 10 cases endocarditis affected a native valve, and only in 2 cases a prosthetic valve. Only 5 patients had underlying valve disease. The most frequently used antimicrobial therapy was the combination of ampicillin plus gentamicin (8 patients). Four cases were treated with monotherapy, 2 with ampicillin, and 2 with vancomycin. Seven patients suffered heart failure, and five of them required surgical therapy with aortic valve replacement. All the patients treated with monotherapy had severe complications, that led to death in 2 of them.
\end{abstract}

KEY WORDS: Endocarditis. Enterococcus. Epidemiology.

Martínez Odriozola P, Muñoz Sánchez J, Arriola Martínez P, Lizarralde Palacios E, Santamaría Jauregui JM, Zuazo Meabe J, Cisterna Cáncer R, de la Villa FM. Endocarditis infecciosa por enterococo: descripción de 12 casos. An Med Interna (Madrid) 2007; 24: 539-542.

\section{INTRODUCCIÓN}

La bacteriemia por enterococo es una infección grave, con unas tasas de mortalidad del $23-46 \%(1,2)$. Una de las complicaciones más importantes de la bacteriemia enterocócica es la endocarditis, que aparece entre el 5 y el $12 \%$ de los casos (1-3). Los enterococos representan la tercera causa más frecuente de endocarditis infecciosa, después de los estreptococos y del Staphylococus aureus, siendo responsables del 5-20\% de todos los casos de endocarditis (4-6). En los últimos años se ha producido un aumento en el número de infecciones producidas por microorganismos del género Enterococcus (2-4) y se han descrito numerosos mecanismos de resistencia antimicrobiana (7). Por otra parte, las infecciones producidas por E. faecium, una especie naturalmente aún más resistente que $E$. faecalis, también aumentan en frecuencia $(7,8)$.

Con el objeto de conocer la incidencia de endocarditis enterocócica en nuestra área sanitaria hemos realizado un estudio retrospectivo durante un período de 12 años, analizando sus características clínicas y microbiológicas, así como el tratamiento y la evolución de la enfermedad.

Trabajo aceptado: 13 de junio de 2007 
TABLA I

CARACTERÍSTICAS DE LAS ENDOCARDITIS POR ENTEROCOCO

\begin{tabular}{|c|c|c|c|c|c|c|c|c|c|}
\hline$N^{0}$ caso & Sexo & Edad & Microorganismo & Válvula & F. desencadenante & Tto. médico. & Tto. quirúrgico & Complicaciones & Muerte \\
\hline 1 & Mujer & 80 & $\begin{array}{c}\text { E. faecalis } \\
\text { (resist.gentamicina) }\end{array}$ & $\begin{array}{l}\text { Mitral } \\
\text { (nativa) }\end{array}$ & No & Vancomicina & No & Insuficiencia cardiaca & No \\
\hline 2 & Hombre & 50 & E. faecalis & $\begin{array}{l}\text { Aórtica } \\
\text { (nativa) }\end{array}$ & No & $\begin{array}{l}\text { Ampicilina + } \\
\text { gentamicina }\end{array}$ & $\begin{array}{l}\text { Prótesis aórtica } \\
\text { mecánica }\end{array}$ & $\begin{array}{l}\text { Embolismos sépticos } \\
\text { Insuficiencia aórtica }\end{array}$ & No \\
\hline 3 & Hombre & 39 & E. faecalis & $\begin{array}{l}\text { Tricúspide } \\
\text { (nativa) }\end{array}$ & ADVP* & $\begin{array}{l}\text { Ampicilina + } \\
\text { gentamicina }\end{array}$ & No & No & No \\
\hline 4 & Hombre & 58 & $\begin{array}{c}\text { E. faecalis } \\
\text { (resist.gentamicina) }\end{array}$ & $\begin{array}{l}\text { Aórtica } \\
\text { (nativa) }\end{array}$ & Prostatectomía & Ampicilina & $\begin{array}{l}\text { Prótesis aórtica } \\
\text { mecánica }\end{array}$ & Insuficiencia aórtica & No \\
\hline 5 & Hombre & 94 & E. faecalis & $\begin{array}{l}\text { Aórtica } \\
\text { (nativa) }\end{array}$ & $\begin{array}{l}\text { Hemorragia } \\
\text { digestiva }\end{array}$ & $\begin{array}{l}\text { Ampicilina + } \\
\text { gentamicina }\end{array}$ & No & No & No \\
\hline 6 & Mujer & 39 & E. faecalis & $\begin{array}{l}\text { Aórtica } \\
\text { (nativa) }\end{array}$ & ADVP & $\begin{array}{l}\text { Ampicilina + } \\
\text { gentamicina }\end{array}$ & $\begin{array}{l}\text { Prótesis aórtica } \\
\text { biológica }\end{array}$ & Insuficiencia aórtica & No \\
\hline 7 & Hombre & 37 & $\begin{array}{c}\text { E. faecium } \\
\text { (resist.vancomicina) }\end{array}$ & $\begin{array}{l}\text { Aórtica } \\
\text { (nativa) }\end{array}$ & ADVP & $\begin{array}{l}\text { Ampicilina + } \\
\text { gentamicina }\end{array}$ & No & Embolismos sépticos & No \\
\hline 8 & Mujer & 67 & E. faecium & $\begin{array}{l}\text { Aórtica } \\
\text { (nativa) }\end{array}$ & No & Vancomicina & $\begin{array}{l}\text { Prótesis aórtica } \\
\text { mecánica }\end{array}$ & $\begin{array}{l}\text { Insuficiencia aótica } \\
\text { Insuficiencia renal }\end{array}$ & Sí \\
\hline 9 & Mujer & 71 & E. faecalis & $\begin{array}{c}\text { Aórtica } \\
\text { (protésica) }\end{array}$ & No & $\begin{array}{l}\text { Ampicilina + } \\
\text { gentamicina }\end{array}$ & No & No & No \\
\hline 10 & Hombre & 42 & E. faecalis & $\begin{array}{l}\text { Aórtica } \\
\text { (nativa) }\end{array}$ & No & $\begin{array}{l}\text { Ampicilina + } \\
\text { gentamicina }\end{array}$ & $\begin{array}{l}\text { Prótesis aórtica } \\
\text { biológica }\end{array}$ & $\begin{array}{l}\text { Insuficiencia aórtica } \\
\text { Insuficiencia cardiaca }\end{array}$ & No \\
\hline 11 & Hombre & 67 & E. faecalis & $\begin{array}{c}\text { Mitral } \\
\text { (protésica) }\end{array}$ & No & Ampicilina & No & $\begin{array}{c}\text { Shock séptico } \\
\text { Insuficiencia cardiaca }\end{array}$ & Sí \\
\hline 12 & Hombre & 57 & E. faecalis & $\begin{array}{l}\text { Aórtica } \\
\text { (nativa) }\end{array}$ & $\begin{array}{l}\text { Hemorragia } \\
\text { digestiva }\end{array}$ & $\begin{array}{l}\text { Ampicilina + } \\
\text { gentamicina }\end{array}$ & No & Embolismos sépticos & No \\
\hline
\end{tabular}

*ADVP: adicción a drogas por vía parenteral

\section{CASOS APORTADOS}

El Hospital de Basurto es un hospital de tercer nivel, de referencia para toda la población de Bilbao (353.162 habitantes en el año 2005). A partir de los registros microbiológicos se han documentado todos los pacientes con aislamiento de enterococo en el hemocultivo y se ha realizado un estudio retrospectivo durante el período comprendido entre el 1 de enero de 1994 y el 30 de abril de 2006, mediante revisión de las historias clínicas de los pacientes diagnosticados de endocarditis infecciosa y de bacteriemia por enterococo e ingresados en los servicios de Medicina Interna, Enfermedades Infecciosas, Cardiología y Cirugía Cardíaca.

Se recogieron los datos epidemiológicos, la puerta de entrada de la infección, los factores desencadenantes de bacteriemia, las manifestaciones clínicas, la comorbilidad, la válvula afectada, las complicaciones, las características microbiológicas, el tratamiento y la mortalidad que presentaron los pacientes. Los datos epidemiológicos incluyeron sexo, edad y la adquisición comunitaria o nosocomial y los microbiológicos la etiología mono o polimicrobiana y la sensibilidad in vitro frente a penicilina, ampicilina, gentamicina y vancomicina. La bacteriemia nosocomial se definió como aquella que no estaba presente en el momento del ingreso hospitalario y que fue adquirida después de 48 horas de estancia en el hospital. Para el diagnóstico de endocarditis infecciosa se emplearon los criterios de Duke (9). El tratamiento quirúrgico de sustitución valvular se refiere a la cirugía realizada durante el ingreso hospitalario, ya fuera realizada con carácter de urgencia o programada. Las tasas de cirugía y de mortalidad incluyeron sola- mente a las que se produjeron durante el mismo ingreso hospitalario.

Durante el período de estudio se diagnosticaron de endocarditis infecciosa un total de 207 pacientes y se registraron 182 casos de bacteriemia por enterococo. Se encontraron 12 casos de endocarditis por enterococo, lo que representó el 5,8\% del total de las endocarditis y el $6,6 \%$ de las bacteriemias por enterococo, con una incidencia anual de 0,28 casos/100.000 habitantes. Se trataba de 8 hombres y 4 mujeres, con una edad media de 58 años (límites: 37-94 años). El microorganismo causante de la endocarditis fue Enterococcus faecalis en 10 casos $(83,3 \%)$ y E. faecium en 2 casos (16,7\%) (Tabla I). Dos de las cepas de E. faecalis eran resistentes a aminoglicósidos y una de las de E. faecium resultó resistente a vancomicina.

La válvula afectada con mayor frecuencia fue la aórtica, con 9 casos $(75 \%)$, seguida de la mitral, con $2(16,7 \%)$ y de la tricúspide, 1 caso $(8,3 \%)$. En el 83,3\% de los casos (10 pacientes) la endocarditis se instaló sobre una válvula nativa y sólo en 2 casos $(16,7 \%)$ sobre válvula protésica. Estos dos últimos pacientes fueron diagnosticados de endocarditis precoz. Únicamente 5 pacientes $(41,6 \%)$ tenían antecedentes de enfermedad valvular cardiaca. No se registró ningún caso de adquisición nosocomial. El 33,3\% de los casos (4 pacientes) tenían enfermedades de base: 1 con carcinoma de colon y 3 con adicción a drogas por vía parenteral (ADVP), 2 de los cuales tenían infección por VIH. El factor desencadenante de bacteriemia pudo encontrarse en 6 pacientes: 3 con ADVP activa, 2 con hemorragia digestiva por tumor de colon (un caso de adenocarcinoma y otro de pólipo adenomatoso) y 1 caso tras resección prostática transuretral. El motivo de ingreso 
fue la fiebre, en 7 casos, síndrome constitucional, 3 casos y anemia normocítica, 2 casos. La manifestación clínica más frecuente fue la fiebre, que estaba presente en todos los pacientes. El tiempo transcurrido desde el inicio de la sintomatología hasta el diagnóstico fue muy variable, oscilando entre los 7 y los 120 días (media 37,5 $\pm 41,1$ ). Entre los datos de laboratorio destacaban la presencia de anemia en el 83,3\% (10/12) de los casos y de hematuria microscópica en el 58,3\% (7/12). Para la realización del diagnóstico por imagen fue necesaria la realización de un ecocardiograma transesofágico en la mayor parte de los pacientes $(66,6 \%)$; el ecocardiograma transtorácico fue diagnóstico únicamente en 4 casos.

El tratamiento antimicrobiano más utilizado fue la combinación de ampicilina y gentamicina durante 42 días, que se empleó en 8 pacientes $(66,6 \%)$. Cuatro casos fueron tratados con monoterapia (por resistencia a aminoglicósidos y/o alergia a beta-lactámicos); 2 de ellos con ampicilina y 2 con vancomicina. Nueve pacientes $(75 \%)$ sufrieron complicaciones, falleciendo 2 pacientes. Siete pacientes $(58,3 \%)$ se complicaron con insuficiencia cardíaca, cinco de los cuales $(41,6 \%)$ precisaron tratamiento quirúrgico de sustitución valvular por desarrollo de insuficiencia aórtica grave. Los 4 enfermos tratados con monoterapia presentaron complicaciones graves, que provocaron la muerte de 2 pacientes (Tabla I).

\section{DISCUSIÓN}

La endocarditis por enterococo es una enfermedad que, en nuestro medio, tiene una incidencia baja y representa el $6 \%$ del total de las endocarditis y el $7 \%$ de las bacteriemias por enterococo, tasas discretamente inferiores a las descritas en otras series $(1,2,4,5)$. Sin embargo, es posible que la incidencia esté subestimada, ya que algunos pacientes de nuestra área sanitaria acuden a otros centros hospitalarios de la red pública o privada. La edad de nuestros pacientes era muy variable y sólo tres de ellos eran mayores de 70 años, a diferencia de lo publicado en otros estudios, que encuentran una mayor frecuencia de endocarditis enterocócica en pacientes ancianos $(4,10,11)$. De hecho, se debe considerar de manera especial el papel de los microorganismos originarios del tracto gastrointestinal en la etiología de la endocarditis en los ancianos, principalmente Streptococcus bovis y enterococo (10). En el lado opuesto, tres enfermos eran menores de 40 años, todos ellos ADVP activos, uno con afección de la válvula tricúspide y dos de la aórtica. La endocarditis enterocócica en ADVP es poco frecuente y se diferencia de la endocarditis estafilocócica por afectar con mayor frecuencia al corazón izquierdo y ser de mayor gravedad (12). Sin embargo, no falleció ninguno de los tres pacientes ADVP, aunque uno de ellos precisó sustitu- ción valvular aórtica.

En nuestra serie, al igual que en lo encontrado en otros estudios, la infección por enterococo tiene predilección por la válvula aórtica, generalmente en pacientes ancianos con valvulopatía degenerativa previa $(4,5,10)$. Únicamente en dos casos la infección se estableció sobre válvula protésica, falleciendo uno de los pacientes. En este estudio no hemos encontrado ningún caso de adquisición intrahospitalaria, a diferencia de lo publicado en otros trabajos que han comunicado una frecuencia del 8-15\% de endocarditis enterocócica nosocomial $(4,13)$. Uno de los hallazgos más llamativos de la presente serie es el importante retraso en el diagnóstico, que podría ser achacable a la ausencia de valvulopatía previa en el $60 \%$ de los pacientes o a las manifestaciones poco floridas de la enfermedad, ya que cinco de los pacientes ingresaron en el hospital para estudio de síndrome constitucional o de anemia.

El E. faecalis fue el microorganismo causante de la endocarditis en la mayoría de los casos, aunque se registraron dos casos de endocarditis aórtica por E. faecium, presentando éstos complicaciones graves y falleciendo uno de ellos. Dos de las cepas de E. faecalis eran resistentes a aminoglucósidos y una de las de E. faecium resultó resistente a vancomicina. No hubo casos de resistencias a ampicilina. El tratamiento combinado con ampicilina y gentamicina fue el más utilizado, lográndose la curación en los ocho pacientes en los que fue utilizado, cinco únicamente con tratamiento médico y tres con tratamiento médico-quirúrgico. Por el contrario, los cuatro enfermos tratados con monoterapia presentaron complicaciones, que condujeron a la muerte a dos pacientes. En general, la mortalidad global de la endocarditis por enterococo es similar a la causada por otros estreptococos, pero menor que la de la endocarditis estafilocócica (4). La mortalidad de nuestros pacientes, que fue del $16 \%$, es similar a la comunicada en otras series $(4,5)$.

\section{CONCLUSIONES}

Para evitar retrasos en el diagnóstico de esta enfermedad se debe tener un alto índice de sospecha, especialmente en pacientes ancianos con patología gastrointestinal o con valvulopatía previa, realizando los hemocultivos de forma precoz. La terapia antimicrobiana de inicio más eficaz es la asociación de un aminoglicósido con un antibiótico que actúe sobre la pared bacteriana (ampicilina o vancomicina).

\section{Bibliografía}

1. Maki DG, Agger WA. Enterococcal bacteremia: clinical features, the risk of endocarditis, and management. Medicine (Baltimore) 1988; 67: 248-69

2. Patterson JE, Sweeney AH, Simms M, Carley N, Mangi R, Sabetta J, et al. An analysis of 110 serious enterococcal infections: Epidemiology, antibiotic susceptibility, and outcome. Medicine (Baltimore) 1995; 74: 191-200.

3. Fernández Fernández FJ, De la Fuente Aguado J, Rubianes González, Pérez Fernández S, Alvarez Fernández M, Nodar Germiñas A, Sopeña
Pérez B, Martínez Vázquez C. Bacteriemia por Enterococcus faecalis. Rev Clin Esp 2004; 204: 244-50.

4. McDonald JR, Olaison L, Anderson DJ, Hoen B, Miró JM, Eykyn S, et al. Enterococcal endocarditis: 107 cases from the international collaboration on endocarditis merged database. Am J Med 2005; 118 : 759-66.

5. Olaison L, Schadewitz K. Enterococcal endocarditis in Sweden, 19951999: can shorter therapy by aminoglycosides be used? Clin Infect Dis 2002; 34: 159-166. 
6. Anderson DJ, Murdoch DR, Sexton DJ, Reller LB, Stout JE, Cabell CH, et al. Risk factors for infective endocarditis in patients with enterococcal bacteremia: a case control study. Infection 2004; 32: 72-7.

7. Fernández-Guerrero ML. Tratamiento de la endocarditis y otras infecciones graves producidas por enterococos. Enferm Infecc Microbiol Clin 1996; 14: 186-91.

8. Noskin GA, Peterson LR, Warren JR. Enterococcus faecium and Enterococcus faecalis bacteremia: Acquisition and outcome. Clin Infect Dis 1995; 20: 296-301.

9. Durack DT, Lukes AS, Bright DK. New criteria for diagnosis of infective endocarditis: Utilization of specific echocardiographic findings. Am J Med 1994; 96: 200-9.

10. Selton-Suty C, Hoen B, Grentzinger A, Houplon P, Maignan M, Juillie- re $\mathrm{Y}$, et al. Clinical and bacteriological characteristics of infective endocarditis in the elderly. Heart 1997; 77: 260-3.

11. Anderson DJ, Olaison L, McDonald JR, Miró JM, Hoen B, SeltonSuty C, et al. Enterococcal prosthetic valve infective endocarditis: Report of 45 episodes from the International Collaboration on Endocarditis merged database. Eur J Clin Microbiol Infecc Dis 2005; 24: 665-70.

12. Reiner NE, Gopalakhrisna KV, Lerner PI. Enterococcal endocarditis in heroin addicts. JAMA 1976; 235: 1861-3.

13. Fernández-Guerrero ML, Herrero L, Bellver M, Gadea I, Roblas RF, De Górgolas M. Nosocomial enterococcal endocarditis: A serious hazard for hospitalized patients with enterococcal bacteremia. J Intern Med 2002; $252: 510-5$. 